\title{
The Microcantilever: A Versatile Tool for Measuring the Rheological Properties of Complex Fluids
}

\author{
I. Dufour, ${ }^{1}$ A. Maali, ${ }^{2}$ Y. Amarouchene, ${ }^{2}$ C. Ayela, ${ }^{1}$ B. Caillard, ${ }^{1}$ A. Darwiche, ${ }^{2}$ M. Guirardel, ${ }^{3}$ \\ H. Kellay, ${ }^{2}$ E. Lemaire, ${ }^{1}$ F. Mathieu, ${ }^{4}$ C. Pellet, ${ }^{1}$ D. Saya, ${ }^{4}$ M. Youssry, ${ }^{3}$ L. Nicu, ${ }^{4}$ and A. Colin ${ }^{3}$ \\ ${ }^{1}$ Université de Bordeaux, IMS, 33400 Talence, France \\ ${ }^{2}$ Université de Bordeaux, LOMA, 33400 Talence, France \\ ${ }^{3}$ Rhodia Laboratoire de Futur, Unité mixte Rhodia-CNRS, Université de Bordeaux, 33608 Pessac, France \\ ${ }^{4}$ Université de Toulouse, LAAS CNRS, 31077 Toulouse, France
}

Correspondence should be addressed to I. Dufour, isabelle.dufour@ims-bordeaux.fr

Received 13 June 2011; Revised 2 August 2011; Accepted 2 August 2011

Academic Editor: Sangmin Jeon

Copyright ( $) 2012$ I. Dufour et al. This is an open access article distributed under the Creative Commons Attribution License, which permits unrestricted use, distribution, and reproduction in any medium, provided the original work is properly cited.

\begin{abstract}
Silicon microcantilevers can be used to measure the rheological properties of complex fluids. In this paper, two different methods will be presented. In the first method, the microcantilever is used to measure the hydrodynamic force exerted by a confined fluid on a sphere that is attached to the microcantilever. In the second method, the measurement of the microcantilever's dynamic spectrum is used to extract the hydrodynamic force exerted by the surrounding fluid on the microcantilever. The originality of the proposed methods lies in the fact that not only may the viscosity of the fluid be measured, but also the fluid's viscoelasticity, that is, both viscous and elastic properties, which are key parameters in the case of complex fluids. In both methods, the use of analytical equations permits the fluid's complex shear modulus to be extracted and expressed as a function of shear stress and/or frequency.
\end{abstract}

\section{Introduction}

Paints, shampoos, gels, and foams are examples of complex fluids we encounter every day. A mesoscopic scale comprised between the molecular scale and the scale of the sample characterized these materials. In the case of foams, it is the size of the gas bubbles. These materials exhibit complex behavior under shear stress, showing both solid and liquid characteristics.

To fully characterize the mechanical behavior of complex fluids, let us consider a small cube of material of section $S$, and let us apply a tangential force $F$ on its top surface. This material is submitted to a shear stress $\tau=F / S$. Under the action of the force $F$, the cube changes its shape. The deformation, which is the ratio of the modified size of the cube minus its initial size divided by its initial size, has two components: an elastic component and a viscous one. In general, the elastic nature of a material is associated with the characteristic equilibrium microstructure in the material. For example, polymeric liquids have a microstructure that is like an assembly of springs representing the linear chains. When this microstructure is disturbed (deformed), thermodynamic forces tend to restore the equilibrium. The energy associated with this restoration process is the elastic energy. Restoration of these springs to their equilibrium state occurs via the release of the elastic energy that was stored during the deformation process. But polymeric fluids are not ideal elastic materials, because they also exhibit viscous dissipation (energy loss) during deformation. When the force is removed, the deformed material undergoes a partial recovery of shape as the elastic energy is recovered; however, the deformation due to the viscous part is permanent. In steady flow, the deformation due to the viscous component increases continuously. Sinusoidal time-varying forces are thus required to probe both components. To this end, the rheological properties of the material are characterized either by a complex viscosity $\eta^{*}$ or a complex shear modulus $G^{*}$ defined by

$$
\begin{gathered}
\eta^{*}=\frac{\tau}{\dot{\gamma}}=\eta^{\prime}-j \eta^{\prime \prime}, \\
G^{*}=\frac{\tau}{\gamma}=G^{\prime}+j G^{\prime \prime}=j \omega \eta^{*}=j \omega\left(\eta^{\prime}-j \eta^{\prime \prime}\right),
\end{gathered}
$$


with $\tau$ being the shear stress, $\gamma$ the shear strain, and $\dot{\gamma}$ the shear rate. Properties $\eta^{\prime}, \eta^{\prime \prime}, G^{\prime}$, and $G^{\prime \prime}$ are, respectively, the real and imaginary parts of the viscosity, the elastic shear modulus (real part of $G^{*}$ ), and the viscous shear modulus (imaginary part of $G^{*}$ ). The "standard" unit of viscosity is Pa.s, but usually, the centiPoise (cP) is used, which corresponds to $0.001 \mathrm{~Pa} . \mathrm{s}$ (the viscosity of water). The unit of the shear modulus is $\mathrm{Pa}$.

This complex notation reveals the possible phase shift between shear stress and shear strain or shear rate. A perfect liquid has a zero elastic modulus $G^{\prime}$, whereas a perfect solid has a zero viscous modulus $G^{\prime \prime}$.

The value of the complex viscosity depends on the amplitude of the oscillation. For small deformations, the response of the system is linear, meaning that the response is additive: the effect of the sum of two small deformations is equal to the sum of the two individual responses. Linear viscoelastic properties are associated with near equilibrium measurements of the fluid. This means that the configurations of the fluids are not removed far away from their equilibrium structures. In this regime, the deformation is proportional to the shear stress, and the shear modulus does not depend upon the amplitude of the applied force. At the opposite end of the spectrum, large applied forces may modify the structure of the sample. In this case, the values of $G^{*}$ depend not only on the frequency of the oscillation, but also upon the amplitude of the applied force. If the excitation frequency is greater than the inverse of the characteristic time of the material, then its structure is frozen, and the material behaves as a solid. Otherwise, if the applied frequency is lower than the inverse of the characteristic time, the structure of the fluid relaxes under shear stress and the material behaves as a liquid.

The classical way to measure the rheological properties of fluids is either to use a viscometer (involving a falling or rollingball) or a rheometer (utilizing the rotational motion of a cone/plate or Couette flow) [1]. Whereas viscometers can only characterize the viscous component of the deformation, rheometers can characterize both elastic and viscous responses. However, the latter have also several drawbacks: the measurement cannot be made in situ (a fluid sample is needed), the amount of fluid necessary to make the measurement is quite large (a few milliliters), and the measurement is limited to low frequency (less than $200 \mathrm{~Hz}$ due to inertial issues).

To overcome the frequency range limitation, some alternative methods have been developed over the two last decades, giving raise to the field of "microrheology," which involves the measurement of the movement of monodispersed beads (microspheres) immersed in the fluid [28 ]. The motion of the beads can be active (optical or magnetic tweezers) or passive (thermal noise) and the acquisition of the motion is performed optically (video particle tracking, laser particle tracking, quasielastic light scattering, and diffusing wave spectroscopy). The major advantage of such methods is the wide frequency range spanned (from $10^{-2} \mathrm{~Hz}$ to $100 \mathrm{kHz}$ ). Unfortunately, as explained in [8], these methods are computationally intensive. In 2007, a particle tracking experiment would require up to 10 minutes of video recording and the analysis of the data would take about 10 hours on a dedicated PC [8]. Even though this limitation becomes less restrictive each year due to increasing computational power, these methods still necessitate timeconsuming procedures. Moreover, for highly viscous fluids, it is challenging to observe the very small motions of the probe particle. Another limitation comes from the fact that if the particle size is less than the mesoscopic scale of the fluid (polymer chains, mesh size, etc.), the measurement yields a local measurement that can be nonrepresentative for the real properties of the bulk fluid.

In order to cover higher frequencies (from $1 \mathrm{MHz}$ to a few 100s of $\mathrm{MHz}$ ), acoustic shear-polarized devices, such as QCM or Love wave devices, have been used [9-14]. These high-frequency devices are very compact, but they also show adverse properties: (i) the penetration depth of the shear wave in the liquid is very low so that only a very thin film viscoelasticity is measured; (ii) the displacement amplitudes are small so that nonlinear effects requiring a certain minimum stimulation in terms of spatial displacements may not become activated and thus are not being sensed. The first item also leads to issues when samples having microstructures extending beyond the penetration depth are investigated, such as may be the case for suspensions and emulsions [15].

In the current paper, we present two alternative methods based on the use of silicon microcantilevers. Both methods are based on the measurement of the hydrodynamic force exerted on a solid body moving in a fluid, which depends on the fluid's rheological properties. In the first method, the moving solid body is a sphere attached to the microcantilever and the force measurement is made using the microcantilever, similar to what is done in AFM systems. In the second method the microcantilever has a dual role: it is used to actuate the fluid flow as well as to measure the hydrodynamic force. Thus, both methods are based on the ability to relate the free-end cantilever deflection to the fluid's rheological properties through analytical equations. Both principles have already been used by the authors [16-20] or other teams [21-33] to measure the viscosity of Newtonian fluids (fluids with constant real part of viscosity and no imaginary part of viscosity). The originality of the presented work comes from the fact that thanks to analytical modeling these methods have been extended to the measurement of the complex viscosity or of the complex shear modulus which characterize both the elastic and viscous behavior of complex fluids.

\section{Method 1: Measurement of the Hydrodynamic Force on a Confined Sphere Using AFM}

2.1. Principle. For this method a glass sphere is glued at the free-end of a silicon microcantilever. In order to have a high hydrodynamic force due to confinement of the fluid (as in a classical rheometer), the sphere is placed near a substrate surface (like the tip of an AFM cantilever) and immersed in the fluid during testing. This setup provides two modes of measurement. 


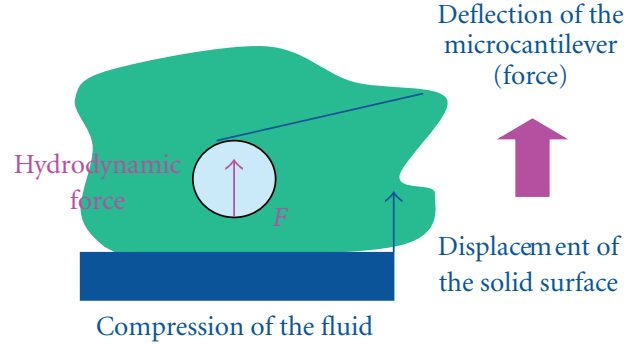

(a)

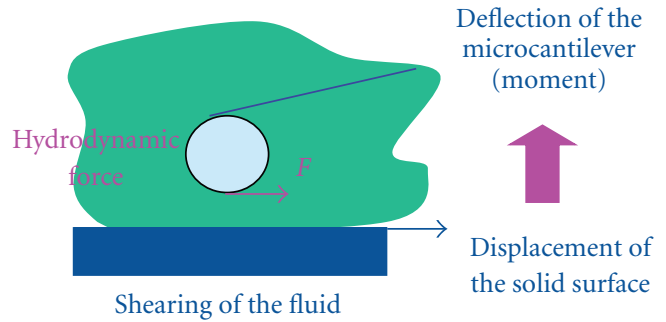

(b)

Figure 1: Principle of the first method. (a) Vertical displacement of the substrate surface and compression of the fluid. (b) Horizontal displacement of the substrate surface and shearing of the fluid.

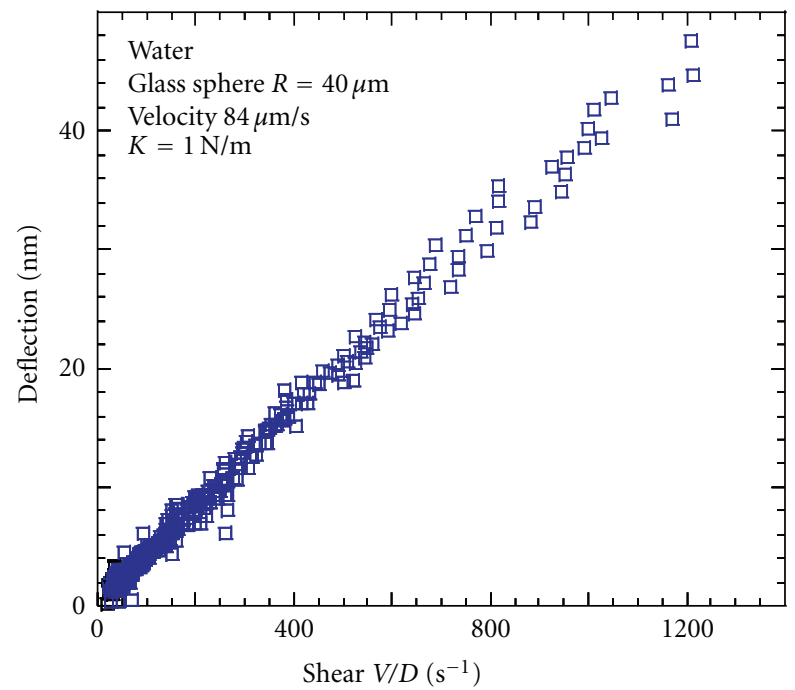

(a)

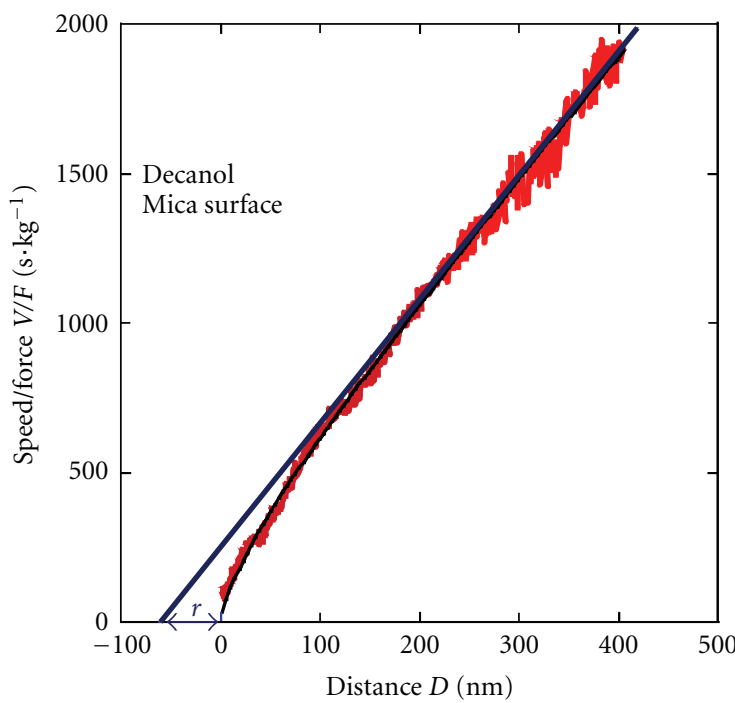

(b)

FIGURE 2: Examples of measurement with Method 1 in configuration of fluid compression. (a) Case of Newtonian fluid, water, without slip (blue squares: measurements). (b) Case of Newtonian fluid, decanol, with slip (red curve: measurements, blue line: fit of the measurement by a line for high distance measurements).

(i) The plane surface is approached towards the sphere (vertical velocity of the plane surface) and the compression of the fluid exerts a hydrodynamic force on the sphere which can be estimated by the measurement of the microcantilever deflection $[25$, 26] (Figure 1(a)).

(ii) The horizontal displacement of the substrate surface shears the fluid (Figure 1(b)). It induces a horizontal hydrodynamic force at the bottom of the sphere [27] which exerts a moment at the free end of the cantilever which results in the cantilever deflection.

In both cases, modeling the fluid-structure interaction allows for the extraction of both the real and imaginary parts of the fluid's viscosity.

To measure hydrodynamic forces, we have used the dimension Veeco AFM in the contact mode. The hydrodynamic force is related to the deflection of the cantilever, which is measured and stored using a 32-bit data acquisition card. Spherical borosilicate particles (GL0186B/106-125, MO-Sci corporation) of $110 \mu \mathrm{m}$ diameter were glued to the free end of a silicon nitride rectangular cantilever ORC8 (Veeco) using epoxy (Araldite, Bostik, Coubert). The vertical displacement of the substrate was induced by a piezoelectric ceramic (Nano-T225, Mad City Labs Inc., Madison, USA).

2.2. Compression Mode. In the case of the compression of the fluid (Figure 1(a)) the hydrodynamic force $F$ can be expressed in the lubrication approximation $(D \ll R)$ by $[20,28-33]$

$$
F=\frac{6 \pi \eta R^{2}}{D} V f^{*}(D)
$$

with $R$ denoting the sphere radius, $D$ the distance between the substrate surface and the bottom of the sphere, $V$ the vertical velocity of the substrate surface, and $f^{*}(D)$ a function that takes into account the slip of the fluid at the surfaces. For the nonslip boundary condition, $f^{*}(D)=1$, so that (2) shows that the microcantilever deflection, as a function of the shear rate, $V / D$, is a line that passes through the origin. The slope of this line depends on the viscosity of the fluid. An example of a measurement is presented in Figure 2(a). 


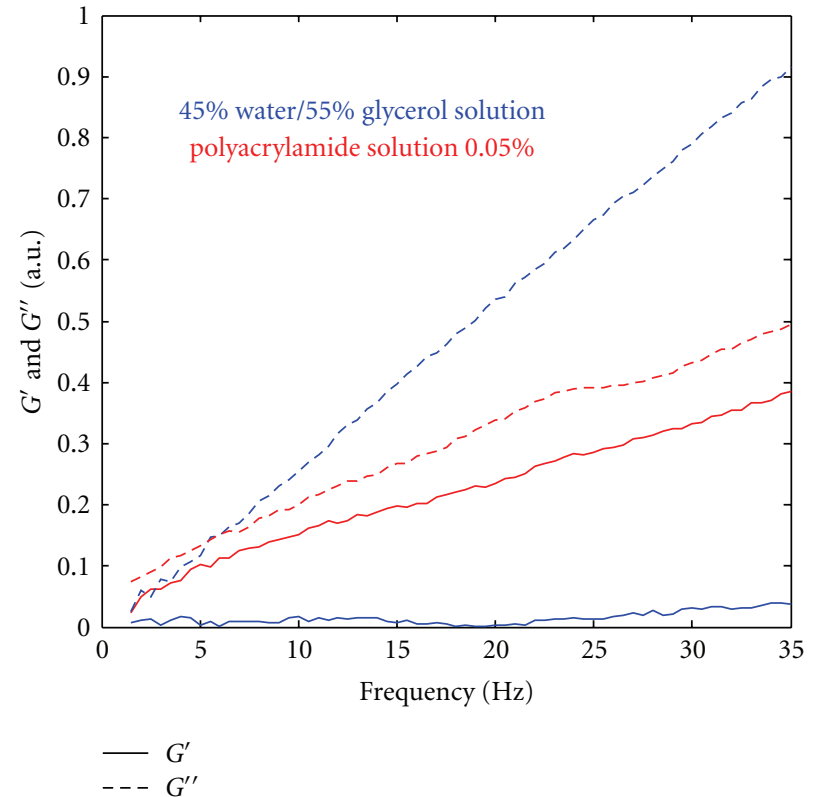

(a)

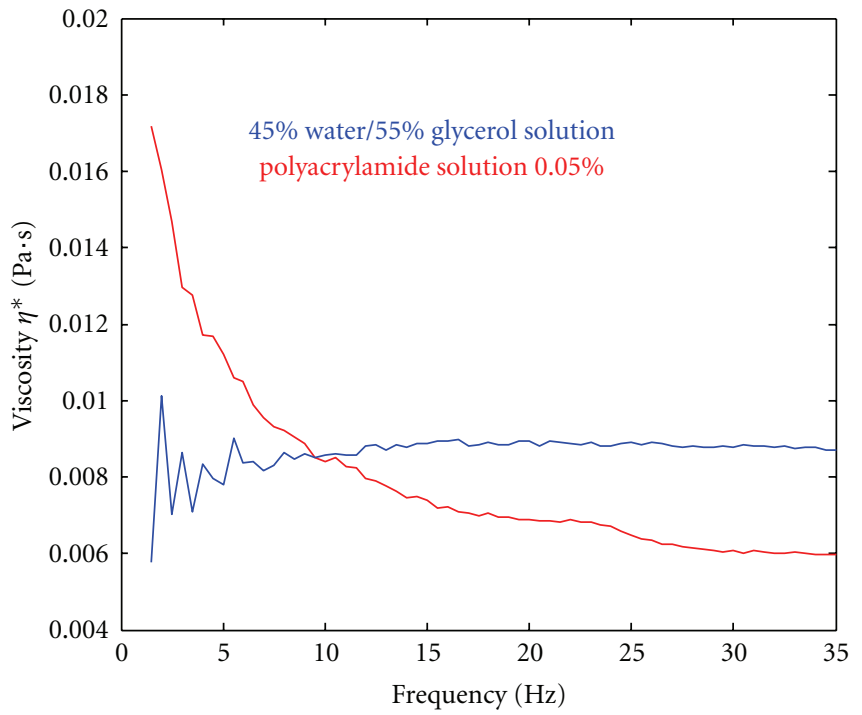

(b)

Figure 3: Example of measurements with Method 1 in shear mode on glycerol/water solution (45wt\%/65wt\%, blue curves) and polyacrylamide solution in water $(0.05 \mathrm{wt} \% / 99.95 \mathrm{wt} \%$, red curves). The distance $D$ is $2 \mu \mathrm{m}$. Shown are the dependencies on frequency for (a) elastic $G^{\prime}$ and viscous $G^{\prime \prime}$ shear moduli and (b) modulus of the dynamic viscosity $\left|\eta^{*}\right|$.

If slip exists at the solid surfaces (substrate and/or sphere), (2) can be simplified for the case where the slip length $r$ is very small compared to the distance $D(r \ll D)$

$$
\frac{V}{F} \approx \frac{D+r}{6 \pi \eta R^{2}},
$$

where $r$ is the sum of the slip-length of the fluid on the two solid surfaces. An example of a measurement utilizing (3) is shown in Figure 2(b): the slip length $r$ corresponds to the distance where the $V / F$ ratio intercepts the abscissa axis.

2.3. Shear Mode. The shear mode (Figure 1(b)) can be achieved by using a piezoelectric ceramic Nano-T225, Mad City Labs Inc., Madison, USA which causes horizontal oscillation of the substrate surface of the form $Y_{\text {piezo }}=$ $Y_{0} \cos (\omega t)$. In this case, both the amplitude $Z_{0}$ and the phase $\Phi_{z}$ of the free-end microcantilever deflection are measured using a lock-in-amplifier (model DSP 7280, AMETEK Inc. Oak Ridge, TN). The free-end deflection is then of the form $Z_{0} \cos \left(\omega t+\Phi_{z}\right)$. Then, the complex viscosity or complex shear modulus can be expressed by

$$
\eta^{*}=\eta^{\prime}-j \eta^{\prime \prime}\left\{\begin{array}{l}
\eta^{\prime}=\frac{G^{\prime \prime}}{\omega}=\frac{Z_{0} \sin \Phi_{z} L_{\mathrm{eff}}}{18 \pi R^{2} Y_{0} \omega \Gamma(D / R)}, \\
\eta^{\prime \prime}=\frac{G^{\prime}}{\omega}=\frac{Z_{0} \cos \Phi_{z} L_{\mathrm{eff}}}{18 \pi R^{2} Y_{0} \omega \Gamma(D / R)},
\end{array}\right.
$$

where $L_{\text {eff }}$ is the effective length of the cantilever, $R$ the radius of the sphere, $Z_{0}$ the amplitude of the transverse deflection of the microcantilever, $Y_{0}$ the amplitude of the horizontal oscillation of the piezoelectric ceramic, $\Phi_{z}$ the phase between microcantilever deflection and piezoelectric oscillation, $\omega$ the radial frequency of the oscillation, and $\Gamma(D / R)$ a hydrodynamic function calculated by Brenner [27] for the case $D \ll R$

$$
\Gamma\left(\frac{D}{R}\right) \approx \frac{8}{15} \ln \left(\frac{D}{R}\right)-0.9588 .
$$

Examples of measurements made with a Newtonian fluid (solution of $45 \mathrm{wt} \%$ water $/ 55 \mathrm{wt} \%$ glycerol) and with a viscoelastic fluid ( $0.5 \mathrm{wt} \%$ polyacrylamide solution) on mica substrate are presented in Figure 3.

These measurements confirm the fact that for the considered frequency range $(1 \mathrm{~Hz}-35 \mathrm{~Hz})$ and shear rate, the glycerol solution is Newtonian whereas the polyacrylamide solution is viscoelastic.

\section{Method 2: Measurement of the Hydrodynamic Force on a Microcantilever Using Deflection Spectrum}

The idea of using the deflection spectrum of a microcantilever immersed in a fluid in order to extract properties of the fluids has been proposed by different authors [2124]. The method is based on the measurement of both the resonant frequency and the quality factor which depend on both the mass density and the viscosity. Depending on the case, the fluid mass density and viscosity are determined either after calibration or by using semianalytical methods. The major drawbacks of such approaches stem from the fact that when using only one microcantilever the viscosity 
is determined at only one frequency, and the elasticity of the fluid is neither taken into account nor estimated. Moreover, a resonant phenomenon is needed in order to measure the resonant frequency and the associated quality factor. Therefore, these approaches cannot be used for highly viscous fluids for which a resonant peak may not exist.

3.1. Basic Principle and Equations for Method 2. When a vibrating cantilever is immersed in a fluid, the fluid exerts a hydrodynamic force on the cantilever due to both the pressure force on the surfaces perpendicular to the cantilever displacement and the shear force on the surfaces parallel to the cantilever displacement. The total hydrodynamic force is composed of two terms: one inertial term proportional to microcantilever acceleration and one viscous term proportional to microcantilever velocity. The microcantilever deflection in the Fourier-space is governed by the Euler Bernoulli equation [34-36]

$$
E I \frac{\partial^{4} w(\omega, x)}{\partial x^{4}}-m_{L} \omega^{2} w(\omega, x)=F(\omega, x)+F_{\text {fluid }}(\omega, x),
$$

where $x$ is the coordinate along the cantilever length, $y$ along the cantilever width and $z$ along the cantilever thickness, $w(\omega, x)$ is the deflection in the $z$ direction at coordinate $x$ and at radial frequency $\omega, E$ is the Young's modulus of the cantilever material, $I$ is the moment of inertia of the cross section at coordinate $x$ with respect to the $y$ axis, $F(\omega, x)$ is the actuation force per unit length at coordinate $x$. In the case of a concentrated force at the free end: $F(\omega, x)=F_{0}(\omega) \delta(x-$ $L), \delta$ being the Dirac function, $F_{\text {fluid }}(\omega, x)$ is the force per unit length exerted by the fluid on the cantilever at coordinate $x$, $m_{L}$ is the mass per unit length of the microcantilever.

The hydrodynamic force can be expressed [37, 38]

$$
F_{\text {fluid }}(\omega, x)=\left(-g_{1}-j \omega g_{2}\right) j \omega w(\omega, x),
$$

with the terms $g_{1}$ and $g_{2}$ representing the viscous and inertial effects, respectively.

According to [39] the following simple expressions may be used to quantify the hydrodynamic force [36]:

$$
\begin{gathered}
g_{1}=\frac{\pi}{4} \rho b^{2} \omega\left(b_{1} \frac{\delta}{b}+b_{2}\left(\frac{\delta}{b}\right)^{2}\right), \\
g_{2}=\frac{\pi}{4} \rho b^{2}\left(a_{1}+a_{2} \frac{\delta}{b}\right),
\end{gathered}
$$

with $\delta=\sqrt{2 \eta / \rho_{f} \omega}$ (fluid layer thickness over which the imposed (cantilever) velocity decays by a factor of $e: 2.72$ ); $a_{1}: 1.0553, a_{2}: 3.7997, b_{1}: 3.8018$ and $b_{2}: 2.7364$.

The solution of (6) depends on the hydrodynamic force which, according to (7) and (8), depends on the fluid density and fluid viscosity.

3.2. Silicon Devices and Examples of Measurements. In order to measure the spectrum of microcantilevers in different fluids, silicon chips have been designed and fabricated (Figure 4). The silicon chips are composed of one cantilever for the measurement and another one as a reference. The

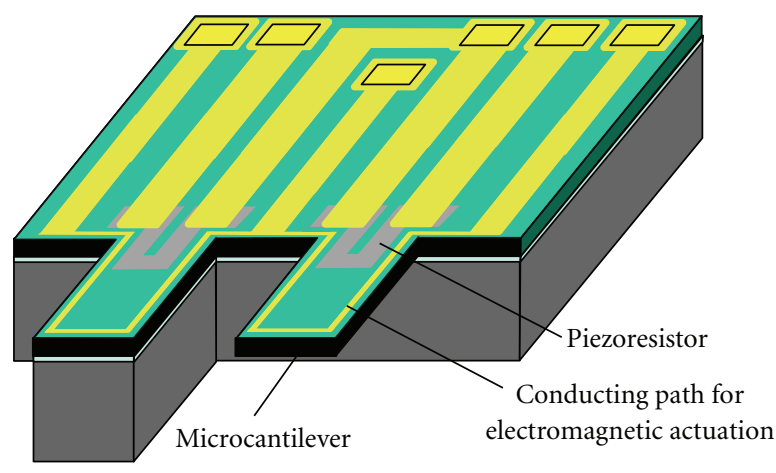

(a)

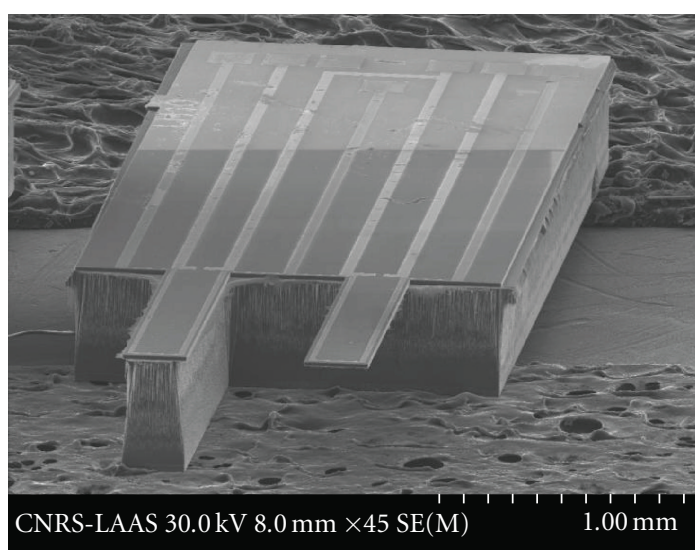

(b)

FIGURE 4: Silicon chips with integrated actuation and measurement designed for measurement of the complex shear modulus as a function of frequency.

TABLE 1: Geometry of the three microcantilevers.

\begin{tabular}{lccc}
\hline Geometry & LL & LH & A \\
\hline Length $(\mu \mathrm{m})$ & 2810 & 1440 & 500 \\
Width $(\mu \mathrm{m})$ & 100 & 285 & 100 \\
Thickness $(\mu \mathrm{m})$ & 20 & 20 & 20 \\
\hline
\end{tabular}

reference cantilever has the same fluidic and electrical environment as the measurement cantilever, but because of its larger thickness (wafer thickness), it does not vibrate. In order to measure the spectrum, actuation and vibration measurement have been integrated. The actuation is an electromagnetic actuation (Laplace force obtained using a sinusoidal current in a conducting path placed on the microcantilever and a static magnetic field created by a magnet placed near the microcantilever). The electrical measurement of the vibration is performed by piezoresistors placed at the clamped-end of the two microcantilevers (the fabrication process is detailed elsewhere [40]).

Three different geometries have been used for the measurements (specimens "LL", "LH", and "A"). They all have the same thickness (SOI wafer) but different lengths and widths (Table 1). 
Vibration measurements have been made with the different microcantilevers in different liquids: water and silicone oils with different viscosities. Examples of spectra measurements obtained using an optical vibrometer (MSA 500, Polytec: the out-of-plane detection mode is based on the Doppler Effect analysis of a laser beam reflection on the cantilever surface) are presented in Figure 5, due to the fact that unsolved electrical coupling issues between the magnetic actuation and the piezoresistive detection have required us to postpone the use of integrated sensing. The measured resonant frequency and quality factor are the same in both measurement systems, but the nonresonant portions of the spectra are quite different. The suppression of the electrical coupling is now under investigation.

As can be seen in Figure 5, the microcantilever spectrum depends on the properties of the surrounding fluid. Moreover, the frequency range for which the spectrum is modified depends on the geometry of the cantilever. It shows that each microcantilever can be used for a specific frequency range: the LL cantilever is for the lowest frequency, the LH cantilever for a low-mid frequency range and the A cantilever (not shown in Figure 5) for a higher frequency range.

3.3. Estimation of the Rheological Properties Using the Microcantilever Spectrum. Using the spectra of the three microcantilevers and (6)-(8) three methods to extract the fluid properties have been developed. They have been called the fitting method (FM), the frequency dependent method (FDM), and the frequency dependent fixed density method $\left(F^{2} \mathrm{M}\right)$. The general principles of these methods and some examples of results are presented hereafter.

3.3.1. Fitting Method (FM). For this method, either the amplitude or the phase deflection spectrum measurement is used. The spectrum measurement is then fitted to a classical second-order low-pass filter response (9) in order to extract the numerical values of both the eigenfrequency, $f_{0}$, and the damping ratio $\xi$

$$
w(\omega, L)=\frac{W_{0}}{1-\left(f / f_{0}\right)^{2}+2 \xi\left(f / f_{0}\right) j},
$$

where $f$ is the actuation frequency $(\omega=2 \pi f)$ and $W_{0}$ the static cantilever free-end deflection.

Then, using analytical equations based on the simplification of the solution of (6) in order to obtain (9), it is possible to calculate the values of the terms $g_{1}$ and $g_{2}$ at the eigenfrequency $f_{0}$. Then, based on (8), two analytical equations can be used to obtain the numerical values of both the fluid viscosity $\eta$ and mass density $\rho_{f}$ from the numerical values of both $g_{1}$ and $g_{2}$ at the eigenfrequency $f_{0}$. The equations for this method can be found in [16].

As an example of the spectra of the LH microcantilever in silicone oils presented in Figure 5, we obtain viscosity values of $11.2 \mathrm{cP}$ and $27.3 \mathrm{cP}$ and mass density values of $790 \mathrm{~kg} / \mathrm{m}^{3}$ and $951 \mathrm{~kg} / \mathrm{m}^{3}$, respectively, for the $10 \mathrm{cP}$ and $20 \mathrm{cP}$ silicone oils. In fact, the viscosity of these silicone oils is temperature dependent. They have been measured at the temperature $19.5^{\circ} \mathrm{C}$ (temperature of the measurement with microcantilever made in a clean room at fixed temperature) using a classical cone/plane rheometer and $10.6 \mathrm{cP}$ and $22.3 \mathrm{cP}$ have been measured.

The advantage of this method compared to the one based on the measurement of the resonant frequency and quality factor [21-24] is that no resonant phenomenon is needed, because the eigenfrequency and damping ratio exist even for high damping and even if there is no resonance. Thus, the fitting method can be used for a higher viscosity range. Moreover, the only required calibration is the measurement of the resonant frequency in air, and all the estimations are based on the use of analytical equations involving no iteration.

The major limitation of this method is that only the fluid viscosity at one frequency per device (i.e., the eigenfrequency of the device in the fluid) is measured. In other words, this method mainly addresses Newtonian fluids.

3.3.2. Frequency Dependent Method (FDM). For this method, both the amplitude and the phase deflection spectrum measurements are needed. Using analytical equations based on the simplification of the solution of (6), it is possible to calculate the values of the terms $g_{1}$ and $g_{2}$ at each frequency of measurement. Using (8), two analytical equations can be used to obtain the numerical values of both the fluid viscosity $\eta$ and mass density $\rho_{f}$ from the numerical values of both $g_{1}$ and $g_{2}$ at each frequency of measurement. The equations for this method can be found in $[17,18]$.

Without calibration and using only the estimated resonant frequencies in air for three cantilevers, the variations of both viscosity and density of $20 \mathrm{cP}$ silicone oil over a wide frequency bandwidth have been calculated as shown in Figure 6. We can see that the viscosity is almost constant over a large frequency range (from $1 \mathrm{kHz}$ to $50 \mathrm{kHz}$ ). The discontinuity between LL and LH results may come from the fact that the method requires an accurate value of the static deflection which is not trivial to be obtained due to low-frequency noise. It can be seen that for higher frequencies $(>50 \mathrm{kHz})$, the viscosity seems to decrease with the frequency, implying shear-thinning behavior, that is, the sample is likely non-Newtonian.

The advantage of this method compared to the FM is that for each device the viscosity is measured over a frequency range and not only for one frequency. The limitation of this method is that the elasticity of the fluid is not taken into account.

\subsubsection{Frequency Dependent Fixed Density Method $\left(F D^{2} M\right)$.} For this method, the first step is exactly the same as for the frequency dependent method: using the amplitude deflection spectrum measurement, the phase deflection spectrum measurement and analytical equations based on the simplification of the solution of (6), the values of the terms $g_{1}$ and $g_{2}$ at each frequency of measurement are calculated. Then, by modifying (8) for the case of a viscoelastic fluid (the viscosity term is replaced by the term $\left.G^{*} /(j \omega)\right)$, two analytical equations can be used to obtain at each frequency of measurement the numerical values of 


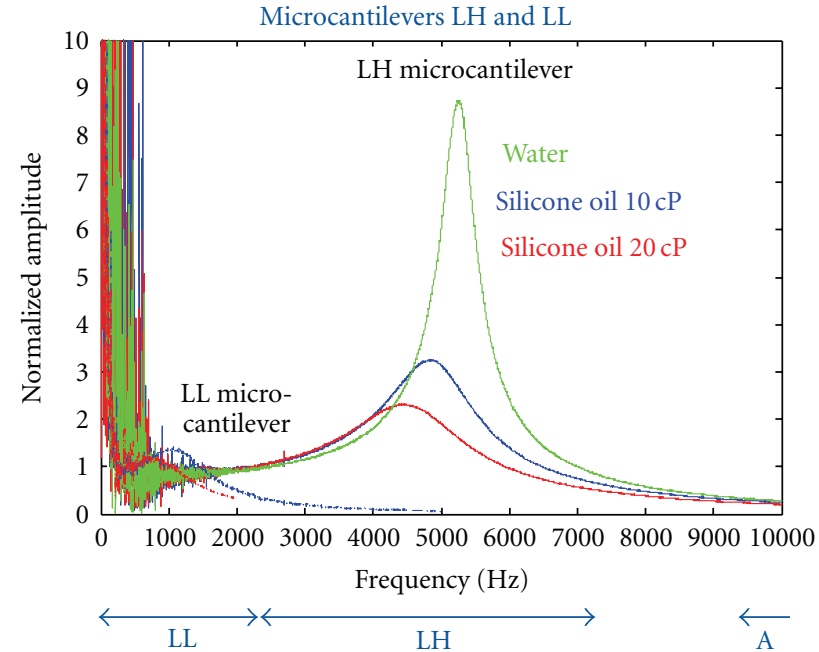

(a)

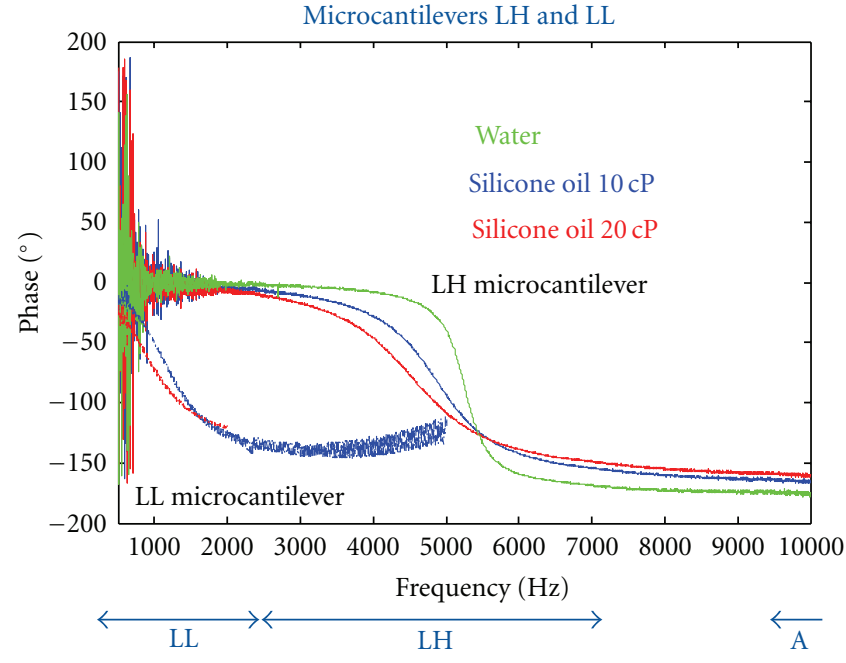

(b)

Figure 5: Examples of measurements of the displacement spectrum of microcantilevers LH and LL in different fluids (water, silicone oils of $10 \mathrm{cP}$ and $20 \mathrm{cP}$ viscosity) at $19.5^{\circ} \mathrm{C}$.

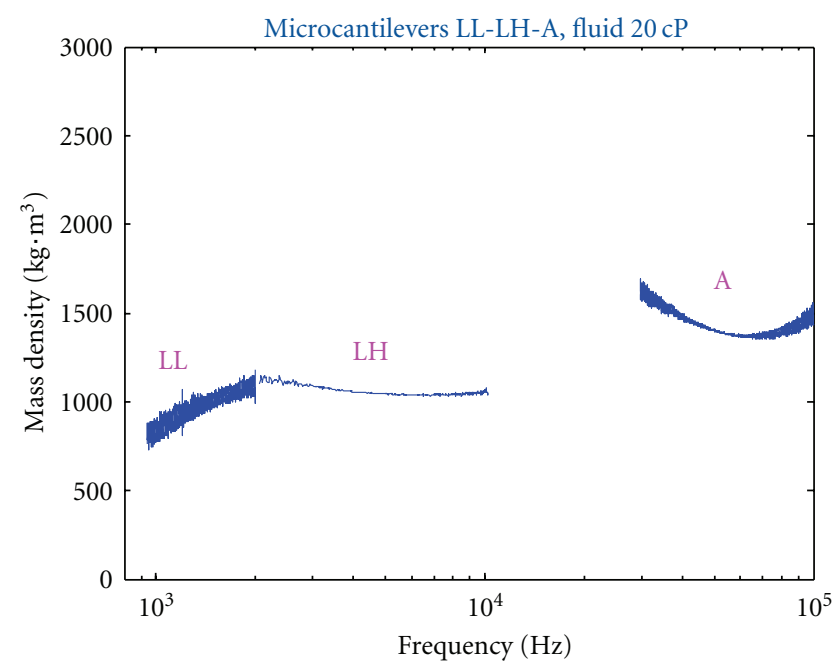

(a)

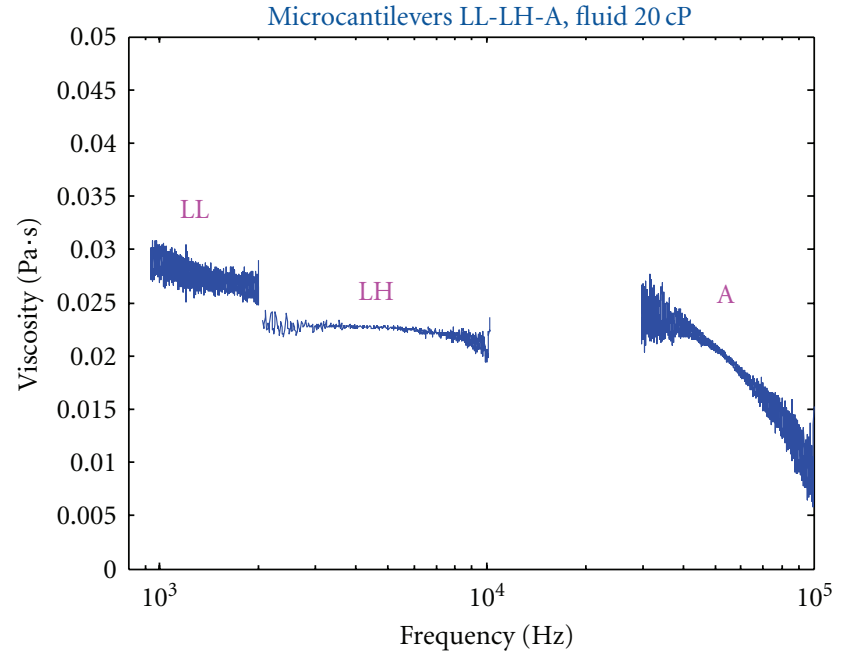

(b)

FIgURE 6: Variation of the mass density and viscosity of the $20 \mathrm{cP}$ silicone oil with frequency using three microcantilevers (LL, LH, and A) applying the frequency dependent method (FDM).

$g_{1}$ and $g_{2}$, from which the real $\left(G^{\prime}\right)$ and imaginary $\left(G^{\prime \prime}\right)$ parts of the fluid's shear modulus may be obtained at each frequency. The equations for this method are given in [19].

Figure 7 shows the variation of viscoelastic parameters of the $20 \mathrm{cP}$ silicone oil over a large frequency range. The low-frequency data were estimated using a classical rheometer of cone-plate geometry. The higher-frequency viscoelastic data could be calculated from the microcantilever deflection spectra (Figure 5) by applying the $\mathrm{FD}^{2} \mathrm{M}$ Method. As can be seen in Figure 7, a feasible continuity between the macro- and microrheological data has been achieved over a large frequency bandwidth although the presence of some missing data arises from the lack of cantilevers of appropriate dimension to cover the missing parts. In general, the viscous $G^{\prime \prime}$ and the elastic $G^{\prime}$ moduli are linearly increasing with frequency and $G^{\prime \prime}$ is always higher than $G^{\prime}$, indicating the viscous nature of the sample up until $50 \mathrm{kHz}$, at which point the moduli display a crossover and $G^{\prime}$ becomes larger than $G^{\prime \prime}$. The Newtonian nature of the sample is well presented by the independence of the complex viscosity of the frequency until $50 \mathrm{kHz}$ beyond which the viscosity exhibits shear-thinning implying the transition to non-Newtonian behavior.

The advantage of this method compared to the FDM is that for each device, both the real $G^{\prime}$ and imaginary $G^{\prime \prime}$ parts of the shear modulus are measured over a frequency range. The limitation of this method comes from the fact that the fluid mass density should be known and that an accurate value of the static deflection has to be extracted from the spectrum measurement. 


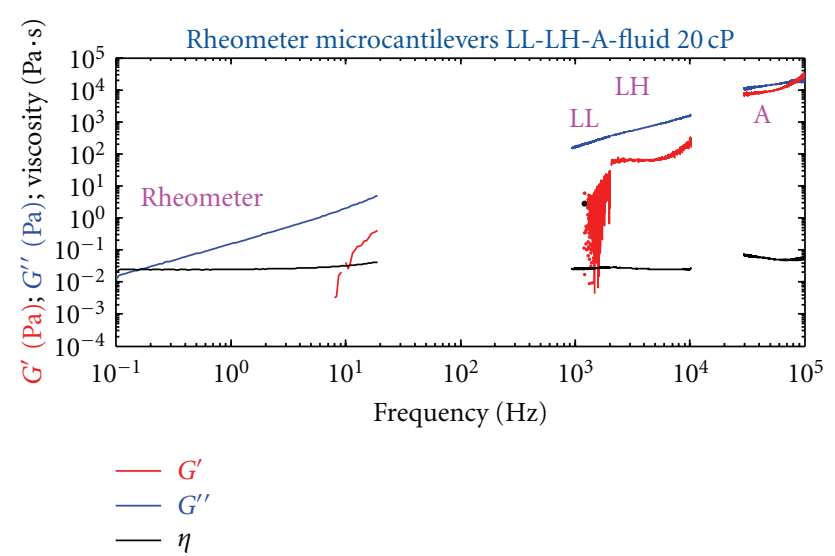

Figure 7: Estimation of the rheological properties $\left(G^{\prime}, G^{\prime \prime}\right.$ and $\left|\eta^{*}\right|$ ) of the silicon oil $20 \mathrm{cP}$ with the three microcantilevers (LL, LH and A) using the frequency dependent fixed density method $\left(\mathrm{FD}^{2} \mathrm{M}\right)$.

\section{Conclusion and Future Work}

Different in situ rheological measurements using microcantilevers have been demonstrated in this paper. With the new extended methods presented herein, only a small amount of fluid is needed to determine the viscoelastic moduli $\left(G^{\prime}\right.$ and $G^{\prime \prime}$ ) of the complex fluids. Depending on the methods, the measurement can be carried out at different frequencies or at different shear rates.

Improvements to the methods are planned for the future with the ultimate goal being to utilize microcantilevers to reliably answer the fundamental question of rheology: "how does matter flow?" For method 1, measurements using textured solid surfaces will be made and the elastic behavior of complex fluids will also be studied by a method using the measurement of the second harmonic of the cantilever deflection which depends on the normal hydrodynamic force exerted on the sphere. For method 2, in order to have measurements at different stress levels as in classical rheometry, measurements under flow conditions will be carried out. Also, many optimizations can be achieved for method 2 as follows:

(i) optimization of the geometry of the cantilevers in order to (i) maximize their sensitivity and (ii) span the $0.5-100 \mathrm{kHz}$ frequency range with no discontinuity,

(ii) investigation of the use of in-plane vibrations instead of out-of-plane vibrations,

(iii) obtain a better understanding and control of the temperature effects [41]; this issue is important due to the very high sensitivity of the calculated values of $G^{\prime}$ and $G^{\prime \prime}$ to any variation of the deflection spectra.

Lastly, in order to deliver easy-to-use microrheometers to rheologists, a complete integrated actuation and detection system must be finalized for all methods.

\section{Acknowledgment}

This work was partially supported by the CPER Pôle $4 \mathrm{~N}$ Nanosciences en Aquitaine (GP-206-action 216/1) with the contribution of the Conseil Régional d'Aquitaine, the FEDER, and the Ministry of Education and Research, by the French National Agency (MicRheo project no. ANR-08NANO-004), and by the Conseil Régional d'Aquitaine (no. 20091102001).

\section{References}

[1] F. A. Morrison, Understanding Rheology, Oxford University Press, New York, NY, USA, 2001.

[2] V. Breedveld and D. J. Pine, "Microrheology as a tool for highthroughput screening," Journal of Materials Science, vol. 38, no. 22, pp. 4461-4470, 2003.

[3] F. C. MacKintosh and C. F. Schmidt, "Microrheology," Current Opinion in Colloid and Interface Science, vol. 4, no. 4, pp. 300307, 1999.

[4] T. A. Waigh, "Microrheology of complex fluids," Reports on Progress in Physics, vol. 68, no. 3, pp. 685-742, 2005.

[5] M. L. Gardel, M. T. Valentine, and D. A. Weitz, "Microrheology," in Microscale Diagnostic Techniques, K. S. Breuer, Ed., Springer, Berlin Heidelberg, Germany, 2005.

[6] N. Willenbacher and C. Oelschlaeger, "Dynamics and structure of complex fluids from high frequency mechanical and optical rheometry," Current Opinion in Colloid and Interface Science, vol. 12, no. 1, pp. 43-49, 2007.

[7] F. Ortega, H. Ritacco, and R. G. Rubio, "Interfacial microrheology: particle tracking and related techniques," Current Opinion in Colloid and Interface Science, vol. 15, no. 4, pp. 237245, 2010.

[8] P. Cicuta and A. M. Donald, "Microrheology: a review of the method and applications," Soft Matter, vol. 3, no. 12, pp. 14491455, 2007.

[9] B. Jakoby and M. J. Vellekoop, "Viscosity sensing using a lovewave device," Sensors and Actuators A, vol. 68, no. 1, pp. 275$281,1998$.

[10] B. Jakoby and M. J. Vellekoop, "Properties of love waves: applications in sensors," Smart Materials and Structures, vol. 6, no. 6, pp. 668-679, 1997.

[11] B. Jakoby, M. Scherer, M. Buskies, and H. Eisenschmid, "An automotive engine oil viscosity sensor," IEEE Sensors Journal, vol. 3, no. 5, pp. 562-568, 2003.

[12] S. J. Martin, G. C. Frye, and K. O. Wessendorf, "Sensing liquid properties with thickness-shear mode resonators," Sensors and Actuators A, vol. 44, no. 3, pp. 209-219, 1994.

[13] S. J. Martin, V. E. Granstaff, and G. C. Frye, "Characterization of a quartz crystal microbalance with simultaneous mass and liquid loading," Analytical Chemistry, vol. 63, no. 20, pp. 22722281, 1991.

[14] D. C. Ash, M. J. Joyce, C. Barnes, C. J. Booth, and A. C. Jefferies, "Viscosity measurement of industrial oils using the droplet quartz crystal microbalance," Measurement Science and Technology, vol. 14, no. 11, pp. 1955-1962, 2003.

[15] B. Jakoby, R. Beigelbeck, F. Keplinger et al., "Miniaturized sensors for the viscosity and density of liquids-performance and issues," IEEE Transactions on Ultrasonics, Ferroelectrics, and Frequency Control, vol. 57, no. 1, Article ID 5361530, pp. 111-120, 2010. 
[16] M. Youssry, N. Belmiloud, B. Caillard, C. Ayela, C. Pellet, and I. Dufour, "A straightforward determinationof fluid viscosity and density using microcantilevers: from experimental data to analytical expressions," Sensors and Actuators A. In press.

[17] N. Belmiloud, I. Dufour, A. Colin, and L. Nicu, "Rheological behavior probed by vibrating microcantilevers," Applied Physics Letters, vol. 92, no. 4, Article ID 041907, 3 pages, 2008.

[18] M. Youssry, B. Caillard, C. Ayela, C. Pellet, and I. Dufour, "Microrheology of newtonian fluids using microcantilever," in Proceedings of the 2nd IASTED International Conference on Nanotechnology and Applications (NANA '10), pp. 540-546, Cambridge, Mass, USA, November 2010.

[19] N. Belmiloud, "Microrheomètre sur puce pour chimie haut débit," Ph.D. thesis, Université Bordeaux, 2008.

[20] A. Maali and B. Bhushan, "Nanorheology and boundary slip in confined liquids using atomic force microscopy," Journal of Physics Condensed Matter, vol. 20, no. 31, p. 315201, 2008.

[21] C. Bergaud and L. Nicu, "Viscosity measurements based on experimental investigations of composite cantilever beam eigenfrequencies in viscous media," Review of Scientific Instruments, vol. 71, no. 6, pp. 2487-2491, 2000.

[22] S. Boskovic, J. W. M. Chon, P. Mulvaney, and J. E. Sader, "Rheological measurements using microcantilevers," Journal of Rheology, vol. 46, no. 4, pp. 891-899, 2002.

[23] M. Hennemeyer, S. Burghardt, and R. W. Stark, "Cantilever micro-rheometer for the characterization of sugar solutions," Sensors, vol. 8, no. 1, pp. 10-22, 2008.

[24] A. Agoston, F. Keplinger, and B. Jakoby, "Evaluation of a vibrating micromachined cantilever sensor for measuring the viscosity of complex organic liquids," Sensors and Actuators A, vol. 123-124, pp. 82-86, 2005.

[25] H.-J. Butt, B. Cappella, and M. Kappl, "Force measurements with the atomic force microscope: technique, interpretation and applications," Surface Science Reports, vol. 59, no. 1-6, pp. $1-152,2005$.

[26] C. Neto, D. R. Evans, E. Bonaccurso, H.-J. Butt, and V. S. J. Craig, "Boundary slip in Newtonian liquids: a review of experimental studies," Reports on Progress in Physics, vol. 68, no. 12, pp. 2859-2897, 2005.

[27] A. J. Goldman, R. G. Cox, and H. Brenner, "Slow viscous motion of a sphere parallel to a plane wall-I motion through a quiescent fluid," Chemical Engineering Science, vol. 22, no. 4, pp. 637-651, 1967.

[28] O. I. Vinogradova, "Drainage of a thin liquid film confined between hydrophobic surfaces," Langmuir, vol. 11, no. 6, pp. 2213-2220, 1995.

[29] R. G. Horn, O. I. Vinogradova, M. E. Mackay, and N. Phan-Thien, "Hydrodynamic slippage inferred from thin film drainage measurements in a solution of nonadsorbing polymer," Journal of Chemical Physics, vol. 112, no. 14, pp. 6424-6433, 2000.

[30] V. S. J. Craig, C. Neto, and D. R. M. Williams, "Sheardependent boundary slip in an aqueous Newtonian liquid," Physical Review Letters, vol. 87, no. 5, Article ID 054504, pp. 054504/1-054504/4, 2001.

[31] E. Bonaccurso, M. Kappl, and H.-J. Butt, "Hydrodynamic force measurements: boundary slip of water on hydrophilic surfaces and electrokinetic effects," Physical Review Letters, vol. 88, no. 7, pp. 761031-761034, 2002.

[32] E. Bonaccurso, H.-J. Butt, and V. S. J. Craig, "Surface roughness and hydrodynamic boundary slip of a Newtonian fluid in a completely wetting system," Physical Review Letters, vol. 90, no. 14, pp. 144501/1-144501/4, 2003.
[33] C. D. F. Honig and W. A. Ducker, "No-slip hydrodynamic boundary condition for hydrophilic particles," Physical Review Letters, vol. 98, no. 2, Article ID 028305, 2007.

[34] S. Timoshenko, Theory of Elasticity, Classic Textbook Reissue Series, McGraw-Hill, 1970.

[35] R. D. Blevins, Formulas for Natural Frequency and Mode Shape, Van Nostrand Reinhold, New York, NY, USA, 1979.

[36] J. E. Sader, "Frequency response of cantilever beams immersed in viscous fluids with applications to the atomic force microscope," Journal of Applied Physics, vol. 84, no. 1, pp. 6476, 1998.

[37] F. R. Blom, S. Bouwstra, M. Elwenspoek, and J. H. J. Fluitman, "Dependance of the quality factor of micromachined silicon beam resonators on pressure and geometry," Journal of Vacuum Science and Technology B, vol. 10, pp. 19-26, 1992.

[38] L.D. Landau, E. M. Lifshitz, J. B. Sykes, and W. H. Reid, Fluid Mechanics, Reed Educational \& Professional Publishing Ltd, 2nd edition, 2000.

[39] A. Maali, C. Hurth, R. Boisgard, C. Jai, T. Cohen-Bouhacina, and J. P. Aimé, "Hydrodynamics of oscillating atomic force microscopy cantilevers in viscous fluids," Journal of Applied Physics, vol. 97, no. 7, Article ID 074907, pp. 1-6, 2005.

[40] D. Saya, T. Leïchlé, J. B. Pourciel, F. Mathieu, C. Bergaud, and L. Nicu, "Contact force control of piezoresistive cantilevers with in-plane nanotips for femtoliter droplet deposition," Microelectronic Engineering, vol. 85, no. 5-6, pp. 1341-1345, 2008.

[41] S. Kim and K. D. Kihm, "Experimental verification of the temperature effects on Sader's model for multilayered cantilevers immersed in an aqueous medium," Applied Physics Letters, vol. 89, no. 6, Article ID 061918, 2006. 

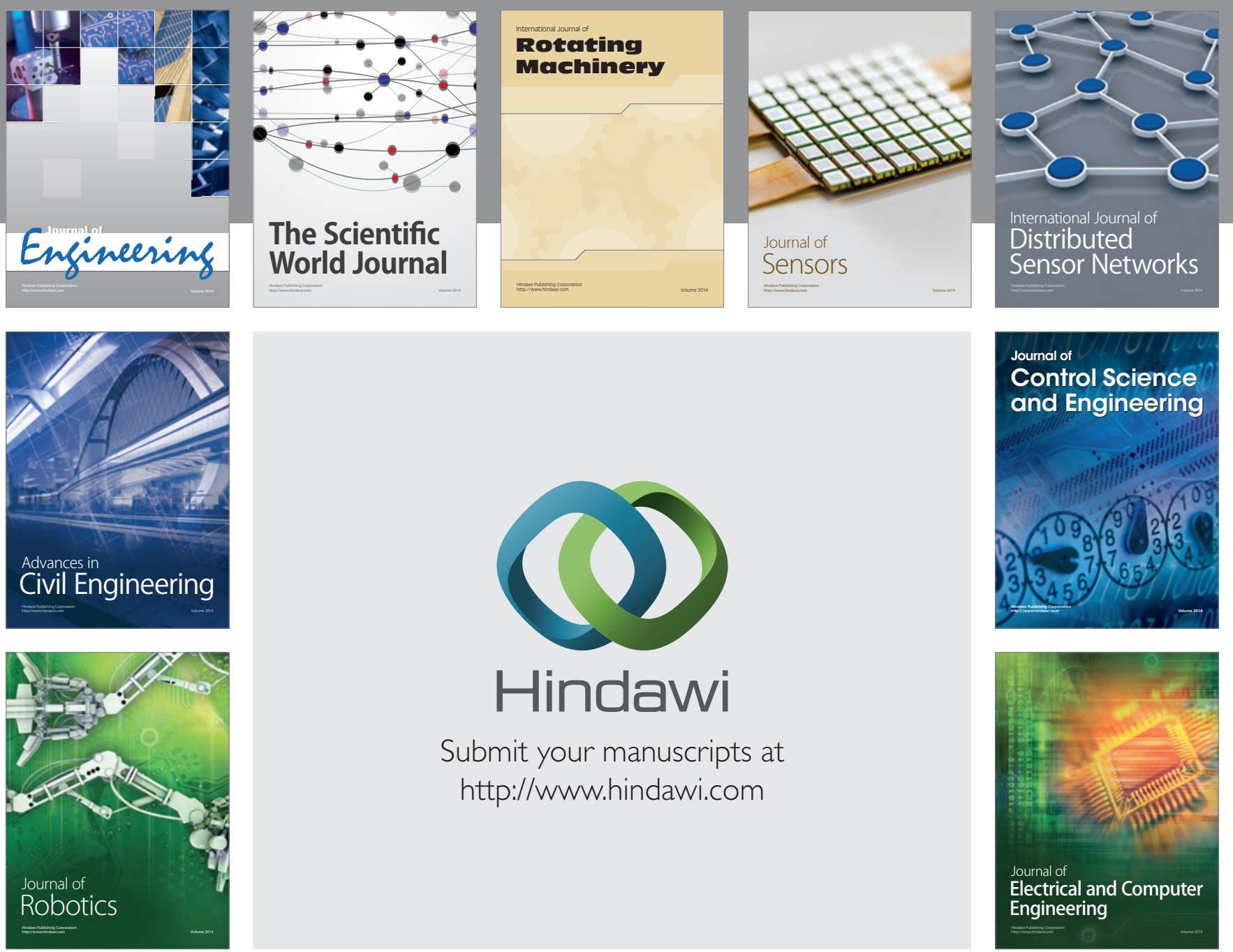

Submit your manuscripts at

http://www.hindawi.com
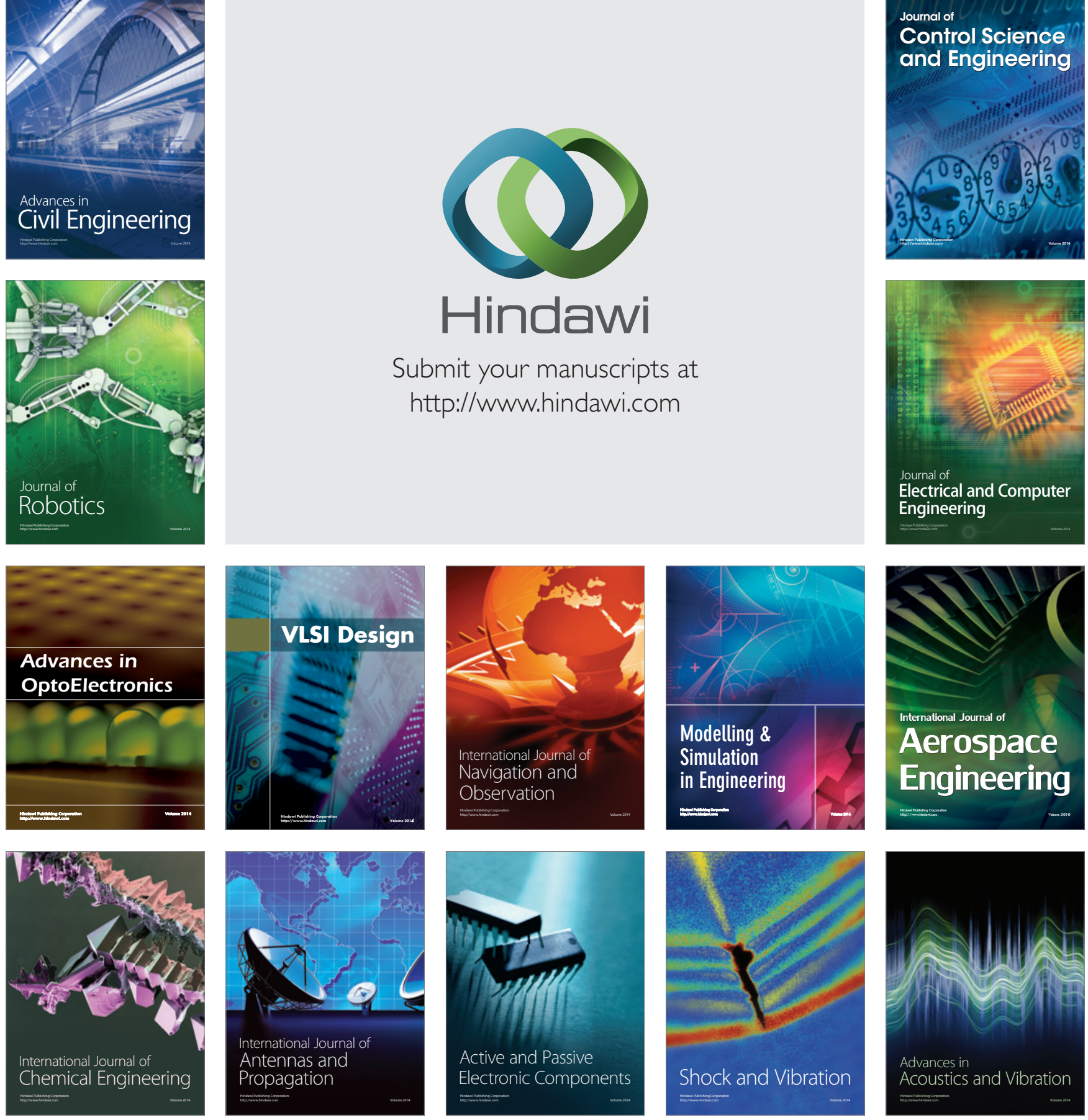